Brit. F. vener. Dis. (1973) 49, 78

\title{
Hospital facilities for, and diagnosis and treatment of, venereal disease in England, 1800-1870
}

\author{
T. J. WYKE \\ Department of Economics and Related Studies, University of York
}

It is apparent, despite gaps in our statistical knowledge, that in the period under consideration the hospital facilities available for patients suffering from venereal disease were inadequate. Depending upon the attitudes of governing bodies, the London hospitals either allocated a number of beds specifically for venereal cases, only admitted cases under special conditions (at the request of the surgeon), or refused admission. The vast majority of those seeking hospital treatment were dealt with as outpatients. By the 1850 s, there is evidence that a number of hospitals, such as the Middlesex and London, relaxed the byelaws which had discriminated against venereal disease, although it is probable that certain venereal cases, the 'innocent sufferers', were allowed treatment regardless of hospital rules. It would, however, be erroneous to view this as a widespread change in attitude towards venereal disease. Samuel Solly, President of the Royal Medical and Chirurgical Society, informed the government committee (Report, 1868: Q 3898) which was investigating syphilis, that he believed the disease was self-inflicted, was avoidable by refraining from sexual activity, and it was 'intended as a punishment for our sins and that we should not interfere in the matter'. Moral smokescreens kindled by the sexual origins of the disease prevented a rational understanding. It was an amalgam of such attitudes which had made John Syer Bristowe and Timothy Holmes, in their official study of hospitals undertaken in 1862 (Report, 1864), dispute the legitimacy of treating venereal patients in voluntary hospitals, since they were not regarded as 'fit objects of charity' and absorbed the slender resources of these financially delicate institutions.

Accepting that the number of beds for patients with venereal disease did increase in the first half of the 19th century, Acton (1857, p. 142) estimated that in 1856 there were only 297 such beds available in the London hospitals (Table). This was to cater for a population of almost 2.5 million and, as many contemporaries sourly complained, compared

Received for publication June 19, 1972 unfavourably with the provision in other European capitals.

The Lock Hospital was the only institution in London specially devoted to venereal patients and it experienced a continual financial struggle throughout the period, relying as it did upon public subscriptions. In 1856, its total income was $£ 1,505-17-9$ and 445 patients passed through its wards, while a further 2,170 were received as outpatients. Indicative of the incidence of the disease was the fact that 8 per cent of the total beds at St. Bartholomew's and almost 10 per cent of the beds at Guy's in 1856 were earmarked for the more difficult venereal cases.

Outside London the supply of beds and the admissions policies were no less varied. In the rapidly growing industrial towns, the practice ranged from hospitals such as the Newcastle Infirmary, where forty of the 215 beds (18.6 per cent.) were allocated to venereal cases, to those such as the Manchester Royal Infirmary which rarely admitted any venereal cases at all, as Manchester had its own 'lock' hospital.

The smaller hospitals displayed a similar diversity. The Salop Infirmary, the Stafford General Infirmary, and the County Hospital at Winchester each had separate 'lock' wards. The South Staffordshire General at Wolverhampton, the Nottingham General, and the county hospitals at Brighton and York were willing to treat venereal patients, usually in the general wards, while the Hereford Infirmary and the West Kent General at Maidstone banned the treatment of venereal cases. The Taunton and Somerset Hospital had similar prohibitive regulations but did in practice admit venereal cases for treatment. Amidst such variety, generalization is dangerous, but it appears that admission was becoming relatively easier in English hospitals, and this especially applied to wives and children who might have unwittingly and unwillingly become infected as well as to patients who were being treated for a different complaint but were discovered also to have a venereal infection.

Before the passing of the Contagious Diseases Acts (1864-69) which provided 'lock' hospitals in the 
TABLE Beds allotted to venereal disease patients in the London Hospitals, 1856

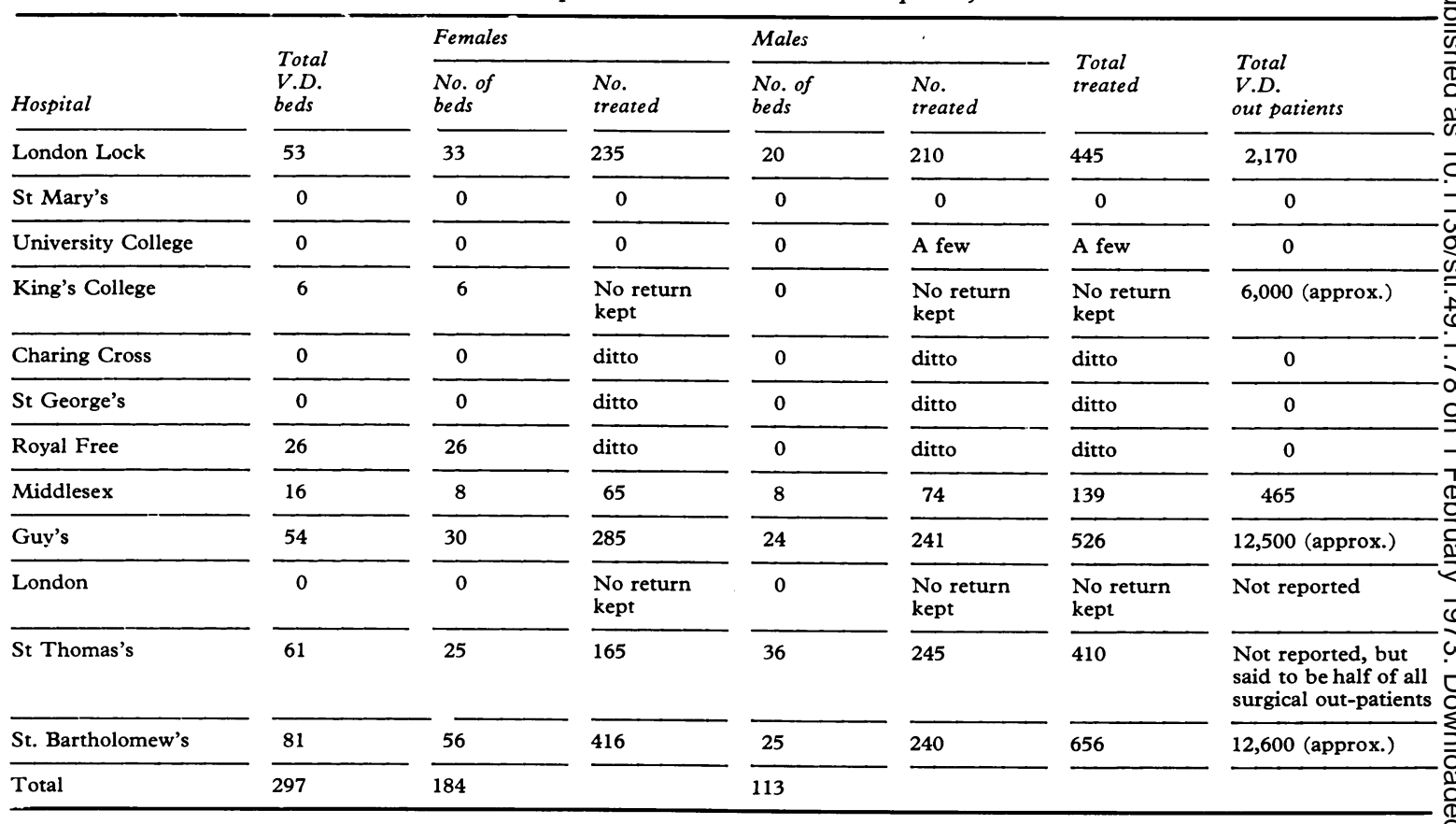

larger naval and garrison towns, it is generally supposed that the only specialist venereal hospital in England was the London Lock. This is, however, to overlook the movements upon the part of the government to establish and finance lock wards in the previous decade. Those attached to the Royal Portsmouth, Portsea, and Gosport Hospital were opened in 1856 and had 28 beds for the treatment of prostitutes. Their operation was, however, far from efficient, for as Bristowe and Holmes (Report, 1864) remarked 'when the arrival of a fleet in the port gives a prospect of gain in their trade, many of the women insist on leaving before their cure is complete'. The Dreadnought Hospital ship, moored off Greenwich, also had the lowest of its three decks assigned for venereal cases.

Calculating the extent of the disease among the civilian population (even neglecting such factors as age, sex, and occupation) is hindered by the dearth of statistics. Hospitals and infirmaries were lax in maintaining both inpatient and outpatient records, while for those who could afford the expense of a private doctor and for the less wealthy and the ashamed who paid for the services of quacks or treated themselves, history is mute. The available figures are thus often little more than the guesses of contemporaries. At a time when the population of Great Britain was over 21 million, T. S. Holland (1854) 'calculated' that there were $1,652,000$ cases of syphilis annually. An

informative sample survey was conducted in August, 1867 , by William Wagstaffe in an attempt to discover the incidence of venereal disease amongst the 'sick poor' of London (Wagstaffe Report, 1869). The numbers of venereal outpatients and inpatients as a pro- $\stackrel{0}{=}$ portion of all outpatients and all inpatients respec- $\bar{\sigma}$ tively at a number of London hospitals revealed the following pattern:

Percentage of patients with venereal disease at 7 London hospitals

Hospital

St. Bartholomew's

St Thomas's

St George's

Royal Free

London

Lambeth Workhouse

St Pancras Workhouse

\begin{tabular}{|c|c|}
\hline $\begin{array}{l}\text { Venereal } \\
\text { outpatients as } \\
\text { percentage of } \\
\text { all outpatients }\end{array}$ & $\begin{array}{l}\text { Venereal } \\
\text { inpatients as } \\
\text { percentage of } \\
\text { all inpatients }\end{array}$ \\
\hline $\begin{array}{l}8 \cdot 14 \\
7 \cdot 08\end{array}$ & \\
\hline $7 \cdot 22$ & $8 \cdot 44$ \\
\hline $17 \cdot 17$ & $\begin{array}{r}28 \cdot 38 \\
4 \cdot 82 \\
2 \cdot 53 \\
10 \cdot 60\end{array}$ \\
\hline
\end{tabular}

Wagstaffe further estimated that 1 in 14 of the poor $\vec{\oplus}$ demanding attention at the medical institutions in $\mathbb{\mathbb { D }}$ London did so for venereal disease.

Recognizing these changes in hospital provision for the civilian population and the government's inter- 8 vention to mitigate the venereal problem in the army 
and navy it may be asked whether similar advances were experienced in the understanding of venereology and the assortment of treatments which were on offer? In one of its periodical reviews of venereal disease, the British and Foreign Medico-Chirurgical Review (1869a) boasted:

'There is perhaps no subject within the range of medical and surgical practice which has made more satisfactory progress during the last half of the century than that of the venereal diseases. It forms one of the points in our science to which we turn with satisfaction when all seems to be in confusion and uncertainty. Here at any rate we are making substantial progress. ....'

However, if one glances at the library of texts and pamphlets on venereal disease in these years, while improvements are apparent, notably in nosology and diagnosis, it is difficult to resist the opinion that the interest in these diseases yielded a small return. In particular, methods of treatment, although challenged, remained virtually unchanged. It was the uncertainty over the subject which caused the government to appoint a committee of enquiry in 1864 . The government held the opinion that 'there is no question in medicine and pathology in which there is such a great diversity amongst medical men of the greatest eminence. They are therefore anxious that the consideration of the committee should mainly be directed to discover a sound principle of treating the disease known under the name of syphilis'.

The 19th century recorded advances in the diagnosis of venereal disease. The Hunterian doctrines were corrected and syphilis and gonorrhoea were recognized as distinct diseases, the results of different poisons. It was, however, on the distinction of various types of syphilis that most medical attention was focused. The Hunterian view of true syphilis, recognized by the induration of the sore and its incurability without mercury, was denied by researchers who demonstrated that the indurated sore was curable without mercury and that it was not this type of sore alone which would result in the familiar timetable of syphilis.

Following the work of Abernethy (1810) and Ricord (1842), the view eventually prevailed that in the primary stage there were two types of syphilitic sore, the true syphilitic sore which affected the constitution and the milder simple sore which did not. The simple sore was characterized by the fact that its influence rarely extended beyond the inguinal glands. It first appeared 3 to 4 days after intercourse as a small pustule; a scab then formed which eventually ulcerated over 15 to 18 days. The lesion was marked by a well-elevated edge which eventually subsided and granulation signalled the healing of the sore. The simple sore was estimated to occur four times as frequently as the true syphilitic sore. Needless to say, accurate diagnosis had important repercussions on treatment.

The true syphilitic sore, regarded as the parent of constitutional syphilis, was recognized as having a variety of forms which doctors began to distinguish. Induration at the base was the cardinal sign, although as the government enquiry made clear this could appear in many forms:

(i) In a cup-shaped ulcer on an indurated base.

(ii) In a shallow abrasion on an indurated base.

(iii) In a deposit of well-defined induration beneath unbroken skin (Report, 1868, p. vii).

From the first two a serous fluid exuded. These sores were believed to have a longer incubation period than the simple sore, of from 15 to 25 days, but it was also thought that this may have been the consequence of a failure on the part of the patient to inform the doctor as they tended to be less painful than the soft sore. As with the soft sore, the inguinal glands became enlarged. The sores were also known to vary in size, texture, and colour depending upon their location and the health of the infected person.

The nagging problem facing venereologists was to decide in the early stages of the disease which sores were simple and which would be likely to result in secondary and tertiary syphilis. The government committee admitted that 'the evidence is conclusive as to the impossibility of pronouncing with certainty upon the character of a sore on its first appearance' (Report, 1868 , p. viii). As numerous medical men were aware, no amount of experience could ensure that the most innocent-looking soft sore would not terminate in constitutional syphilis. Some 4 to 10 weeks after the primary sores had disappeared, the secondary stage commenced. The distinctive features were a feeling of lassitude and depression, aching in the joints and limbs, and generalized eruptions, usually beginning as pale pink before turning a copper colour; the throat and mouth also became affected. At this stage the disease was held to be non-infectious.

The symptoms subsided after a 'number of months' and there followed a period of quiescence of an indeterminate length before the tertiary symptoms appeared. At this stage it was recognized that the venereal poison had invaded the tissues of the body; and the brain and liver were particularly liable to be attacked, and ultimately the disease could result in mania, epilepsy, paralysis, and even death.

A major feature in this unravelling of the venereal diseases was the attempt to replace diagnosis by sight and touch with the more accurate method of inoculation. Here the seminal influence was Philippe Ricord (1842), who pursued diagnostic tests by 
inoculating venereal pus into diseased patients and recording the results. Ricord also assisted diagnosis by detailed descriptions of venereal sores at different stages of development. In England, it is difficult to assess how many doctors used this process. Henry Lee (1863) was one of the more scientific exponents of inoculation and achieved important results when he demonstrated that the true indurated sore was not auto-inoculable. Hostility towards inoculation, however, was apparent amongst English doctors. Edward Cutler (Report, 1868: Q 4129) related an example of an inoculated patient whose resulting sores took months to resolve. He believed that inoculation was 'a practice that is quite wrong ... that it produces a vast deal of mischief, that it is a practice which is perfectly unnecessary, and that it does great injury to the patients'. By 1870 there were also such a large number of exceptions to the general rules of inoculation laid down by Ricord that it could not be relied upon as an accurate test. It is highly probable, that in England the majority of doctors, unless serving in a specialist hospital, would have remained dependent upon clinical observation. It is also worth noting that in diagnostic practice little use was made of the microscope.

Concerning the method by which the disease was transmitted, there was more general agreement that the poison either entered the system through a minute wound caused before or during coitus or that the poison remained in the folds of the genital membranes and was absorbed into the system. The latter course explains the emphasis which doctors placed upon ablution as soon as possible after intercourse. The transmission of syphilis to innocent parties, especially hereditary syphilis, was also recognized.

Reflecting the diversity of opinion over pathology and diagnosis was the variety of methods of treatment recommended for syphilis, dependent on the type and stage of disease diagnosed. The chief weapon in the materia medica armoury was mercury which could be administered internally in the form of a pill, or externally as an ointment or in the form of a vapour bath. Experiments with hypodermic injections were not undertaken until the $1860 \mathrm{~s}$. Many pills were available, the most popular being the blue pill made from mercury, confection of roses, and powdered liquorice. Belloste's pills were concocted from mercury, aloes, rhubarb, scammony, and black pepper, while Sedillot's pills were composed of mercurial ointment, medicinal soap, and powdered marshmallow (Lancereaux, 1869). In spite of improved diagnosis there was much evidence that mercury was injudiciously administered with frightening consequences. Rule of thumb methods prevailed. William Acton explained his method of application to the government enquiry in the following terms: ' $I$ almost invariably use inunctions on the inside of the knee. I $\overline{\bar{C}}$ take a portion of mercurial ointment as large as the $\vec{\nabla}$ top of my thumb and rub it in' (Report, 1868: $\unrhd$ $Q$ 3015). Dosage was in fact controlled by reference $\%$ to the effect of the mercury on the venereal symptoms $\overrightarrow{0}$ or until signs of mercurial poisoning were evident. These guidelines were sanctioned by the witnesses $\vec{\omega}$ before the government investigation. Boerhaave $\frac{O}{o}$

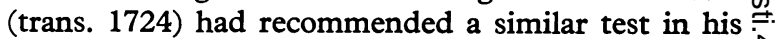
widely-consulted medical aphorisms 150 years earlier: 'Aphorism 1476

As soon as the breath begins to stink, the gums to ake, $\infty$ the teeth to grow loose and stick out, then ought the $\frac{\text { o }}{3}$ physician to attend carefully and consider whether he ought to go on, to stop, or even make some diversion.' T

Because the remedy was often worse than the $\frac{D}{2}$ disease and because some sores were stubborn under mercurial treatment, doctors sought other remedies. The list of alternatives was long, and in 1800 John $\vec{\varphi}$ Pearson, surgeon to the Lock Hospital, catalogued $\omega$ some of the popular medicines; these included lignum $\square$ guaici, sarsparilla, cinchona, opium, cicota, walnut, ammonia, sulphuric acid, and nitric acid (Pearson, 1800). Possibly the most important addition to the therapeutics of syphilis was the introduction of $\frac{O}{D}$ iodide of potassium by Wallace (1836). This became a popular antisyphilitic, without the hazardous consequences of mercury, and was particularly recommended for use in the tertiary stage of the disease.

In the early decades of the 19 th century a strong reaction set in against mercury and doctors began to favour the non-mercurial plan of treatment. The 'simple method', as it was designated, consisted in treating primary syphilis with a strict regime of rest, $\stackrel{\circ}{\circ}$ cleanliness, and controlled diet. This was combined with the use of antiphlogistics and bloodletting. The 을 treatment had resulted from the observations of $\supset$ English medical officers serving in the Peninsular War, and the initial researches of Fergusson (1813), N Rose (1817), and Guthrie (1817) found eager fol- os lowers on the continent where the treatment was $N$ popularized by Desruelles and Jourdan. This rejec- N tion of mercury was not a new idea as various doctors from the 16th century onwards had favoured other 0 medicinal agents. In fact, in the years 1770-1830, a steady stream of publications from medical men such $\stackrel{?}{+}$ as Andrew Duncan (1772), Andrew Mathias (1810), 0 and James Hamilton (1819) had issued caveats against the use of mercury.

Mercury was not, however, as widely rejected in $\mathbb{\mathbb { D }}$ England as on the continent. English doctors still $\bar{\sigma}$ preferred to dose, poultice, and purge their patients rather than to do nothing. Simple sores, when identified, were treated by local applications and not 
by mercury, a powerful caustic such as nitric acid being applied to burn the sore. Other doctors preferred to use the scalpel and excision. For the true indurated sores and secondary stage of syphilis mercury remained indispensable. As the British and Foreign Medical Review (1840a) concluded:

'The universal tendency of modern experience is in favour of the mercurial treatment of this form of disease, and although this as well as the other forms of primary syphilis may get well without mercury, still the cure is uncertain and prolonged, and generally leaves behind it a hardened cicatrix, prone to ulcerate again.'

A similar note was echoed in the government enquiry, but caution was still necessary to ensure that only 'moderate ptyalism' occurred and, while the government committee saw no reason to comment on the indiscriminate use of mercury, other medical men frequently observed the sad consequences of overdosing. To quote the British and Foreign MedicoChirurgical Review (1869):

'We are so familiar with the injury which may arise from a misuse of the so-called specific remedies, that we are all on our guard, and never give them except with good reason, and under proper precautions.

'Is there any rule to guide us in the treatment of syphilis by specifics or otherwise? In any given case can we say with certainty whether mercury should be used or iodide of potassium, or whether the case will do equally well without either of these remedies? To this we must answer there is no absolute rule of this kind.'

In such a situation cures were not guaranteed, there was the constant fear of relapse, and doctors advised patients who were considering marriage to postpone it for at least one year. It is not difficult to understand why quack remedies flourished.

As with so many medicines, a greater ignorance was apparent when the question of how in fact mercury operated against syphilis was asked. Some doctors used mercurial treatments but were baffled by its action. $\mathrm{Mr}$ W. S. Savoury, Assistant-Surgeon at St Bartholomew's Hospital, gave the following evidence to the government enquiry:

' $Q$. What is the action of mercury upon the syphilitic disease?

A. I have not the slightest idea in the world. I do not know that $I$ understand what the modus operandi of any medicine at all is.' (Report, 1868: Q 5521)

A common notion was that the mercury neutralized the venereal poison in the blood as alkali acts on acid, the inference being that the sooner one saturated the system with mercury the better were the chances of recovery. Others saw mercury not as an antidote but as a catalyst which quickened the train of symptoms. The failure to understand the action of mercury reflected the relative backwardness of therapeutics in the medical sciences. In spite of these areas of ignorance, in the absence of other effective forms of treatment for primary and secondary syphilis, mercury remained, in England, the general panacea, although continental doctors had become increasingly eclectic in their treatment of the disease.

Gonorrhoea was regarded with less concern than syphilis. In the male it was characterized by inflammation of the urethra, resulting in pain on passing water, and a yellowish-white discharge and sometimes a swelling of the testicles. It was recognized that the external symptoms were less obvious in the female and while an increased discharge, soreness in the vagina, and difficulty in passing water would be suspicious signs, only a thorough examination could settle the question. The vagina was believed to be the usual seat of the infection, although the labia, clitoris, and urethral meatus might also be attacked.

Solutions and lotions were increasingly used in treatment; mercury was regarded as a dangerous and ineffective therapy, but was still used in the first half of the century despite the warnings of Sir Astley Cooper (Waugh, 1971). The British and Foreign Medical Review (1840b) took William Wallace, surgeon to the Jervis Street Infirmary in Dublin, to task for using mercury in the treatment of gonorrhoea.

'... we find Dr. Wallace, on the theoretical assumption of the identity of the poisons in gonorrhoea and syphilis, recommending five grains of blue pill with a quarter of opium, twice a day for two or three weeks.'

For the male, a diversity of treatments was advised. An initial step was to steep the penis in hot water two or three times a day as soon as the discharge appeared. Powerful solutions (often of nitrate of silver in distilled water) were instilled into the urethra, and some surgeons applied caustics with a bougie. Leeches were also applied to the infected parts to reduce the swelling. Many doctors relied on doses of copaiba, balsam, or cubebs, although this often made the patient sick. For the female, treatment was also largely dependent on frequent instillations and ablutions with solutions of nitrate of potash. Doses of copaiba were again recommended. In acute cases cauterization was performed. Dissatisfaction with the results fostered ingenuity in devising new treatments. Dr Alexander Hannay of the Glasgow Lock Hospital revealed in a communication to the editor of the London Medical Gazette that experimental treatments could have unforeseen consequences. Outlining his methods he wrote:

'I introduce a stick of nitrate of silver into a quill, and tie a thread firmly around the lower part of the quill to fasten the caustic, which I leave projecting beyond the quill by about half an inch. I generally smear the quill with a little lard and introduce. I then deliberately and 
slowly withdraw it, turning it round so as to bring it in as extensive contact as possible with the lining of the vagina. I may add that, by accident, the nitrate of silver has more than once broken in the vagina, and could not be found. It caused me much alarm and anxiety at first ... and though I would carefully avoid it, I now regard the occurrence as of very little importance.' (Hannay, 1837)

As with syphilis, the disease was generally pronounced 'cured' with the disappearance of the external symptoms.

One of the more startling therapeutic episodes, which reflected not only the dissatisfaction of the medical profession with existing treatments but also their attitudes and ignorance towards venereal disease, was the attempt to provide immunity from syphilis as well as treatment by continual inoculation of the venereal virus. The immunity was characterized by an absence of sores and chancres and the constitution was thought to be no longer capable of being affected by syphilis. Joseph Alexandre AuziasTurenne presented his theory in 1850 to the French Academy of Medicine, where it was acrimoniously condemned, not for empirical reasons (though admittedly there was a shortage of case studies) but on the grounds that it was unethical to provide a prophylactic against syphilis. The British and Foreign MedicoChirurgical Review (1857a) initially supported the decision of the French institution to stop further research:

'We cannot deny that they had right on their side; this proposal was not only immoral, for the disease is one to which an individual voluntarily submits himself by a lapse from the rules of morality, but it was also most injudicious to subject a perfectly healthy person to the dangers of incurring a malady from which he might never again be able to free himself.'

The vehemence and indignation of the arguments against the 'syphilizers', as they were called, was often at the expense of logic and clarity, but the conclusion was self-evident: for the majority of doctors the venereal diseases were open to treatment but not to prevention. When prevention was discussed it centred in the sanitary inspection of prostitutes and the need to encourage ablutions, not the possibilities of schemes of immunization.

This denial of the freedom to experiment did not, however, dissuade other doctors, and more systematic 'syphilization' experiments were carried out in the 1850s, chiefly by William Boeck of Christiania and Sperino of Turin. It was significant that they felt it necessary to justify their research on the grounds that they regarded 'syphilization' as an alternative to mercurial treatment and that they spoke increasingly less of its preventive properties.

'The great question in our opinion is in what cases should syphilization be employed ? As a prophylactic its adoption is unjustifiable, and even its discoverer now holds this opinion. Syphilization can therefore only be adopted where venereal disease already exists. . . ' (Boeck, quoted $\overline{\bar{\rho}}$ in the British and Foreign Medico-Chirurgical Review $\mathbb{\Phi}$ (1857b)).

The actual method of treatment was both painful के and prolonged. Numerous inoculations were made $\vec{\circ}$ on the chest, arms, and legs of the patient, initially $\overrightarrow{\vec{H}}$ using matter from the syphilitic ulcer of another $\mathscr{\omega}$ patient. The procedure was continued until the mat- of ter was no longer inoculable-this could be from 3 to 6 months. Such experiments were not the kind suited to the heroic gestures of self-exposure to danger by medical men and many of the guinea-pigs were ${ }_{0}^{\infty}$ prostitutes. The results, however, appeared to justify the continuation of the research; Boeck reported that $\vec{T}$ primary and secondary syphilis were cleared and that $\mathbb{D}$ there was an insignificant rate of relapse. As with mercury, the missing piece of the puzzle was the precise modus operandi of 'syphilization'. Not sur- $\overrightarrow{-}$ prisingly, erroneous analogies were made with smallpox vaccination, but pathological knowledge was $\omega$ lacking and this treatment remained empirical.

The cautious attitude of British medicine to 'syphilization' held back testing of the treatment $\bar{\partial}$ until William Boeck on a visit to England persuaded 气

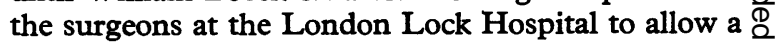
trial. The tests were initiated by Boeck and then con- $\overrightarrow{\vec{B}}$ tinued by James Lane and George Gascoyen (1867) $\frac{0}{3}$ in the cases of 27 patients. There was a high rate of $\vec{F}$ recovery but Lane and Gascoyen disagreed whether this was due to the treatment. Lane believed that 'syphilization' had been beneficial to the patients and resulted in less liability to relapse, while Gascoyen 3 attributed the improvement to the regular diet and rest in the hospital. Despite these differences they $\frac{0}{3}$ both firmly concluded that the treatment was not 0 appropriate:

$\therefore$... syphilization is not a treatment which can be $\frac{7}{0}$ recommended for adoption. We consider that, even if it could be permitted to possess all the advantages claimed $\mathrm{N}$ for it by its advocates, its superiority over other modes of treatment, or in many instances over no treatment at all, $P$ would not sufficiently compensate for its tediousness, its $\mathrm{\omega}$ painfulness, and the life-long marking which it entails upon the patient.'

Little sympathy was thus found for 'syphilization'; $\frac{\mathrm{C}}{\mathbb{D}}$ there had been an unwillingness to undertake re- ? search and after an uneasy flirtation with the system $\square$ those medical men who were interested were possibly $\bar{O}$ satisfied with the conclusions of Lane and Gascoyen. In retrospect, the behaviour of the English medical $\frac{\mathbb{Q}}{2}$ profession can be recognized as the result of conserva- $\bar{\sigma}$ tism and of a prejudice fixed in the immorality of $?$ immunization. English physicians preferred to응 condemn in ignorance and would have seconded 
the graphic warnings of the British and Foreign Medico-Chirurgical Review (1865):

'Woe be to the wretch who falls into the hands of a believer of syphilization! We had occasion to see several times both in Paris and Vienna, patients who had been thus treated, and whose arms, back, chest, and legs were pitted with innumerable cicatrices. Nor, shall we easily forget one 'miserable' who lay with an enormous phagedaenic sore, involving the whole of one buttock and the upper part of the thigh-a consequence of inoculation.'

One of the factors which may help to account for slow progress in diagnosis in these years is the slow adoption of instruments to assist the examination of the genitals. The speculum was the important development and although it was widely used on the continent it met with a guarded acceptance in England. Again the practical utility of the technique was swamped by expressions of moral disapproval. Attempts to encourage the use of the speculum by such individuals as Balbirnie (1836) were rebuked. The speculum examination was regarded as a traumatic experience and concern for the delicacy and modesty of the patient was placed before accurate diagnosis. It was thought to be

'. . very doubtful what amount of good can compensate a young girl, or even a wife, for being placed upon a bed or table, her thighs held wide asunder, a speculum thrust into her vagina, and screwed open until all within is exposed, while a man is curiously looking in, by the light of the sun or of a tallow candle. ... Its employment may be painful, and is disgusting. It is highly disagreeable to the surgeon or physician, and must be revolting to every woman of delicacy. Strong measures only, in any particular cases, can justify its use upon modest females.'

Moreover a less offensive form of diagnosis was available, and the traditional method was preferred:

'The character of the discharges and the general symptoms will usually enable one to determine whether the uterus is affected. When they are insufficient we possess a means of examination which, in the great majority of cases, is quite as satisfactory as the employment of the speculum, and possesses not the one-hundredth part of its indecency. We mean, of course, examination by the finger.' (MedicoChirurgical Review, 1837)

This attitude continued despite the acknowledgment that in women the venereal symptoms were less prominent. Hunter had been aware of this problem and Robert Williams (1841) opined that 'no more difficult question exists in medicine, than to determine, whether they are or are not affected'. Little evidence is available on the rate of adoption of the speculum (the same may be said of the thermometer, stethoscope, ophthalmoscope, and hypodermic syringe), but it is perhaps significant that Acton (1857) believed that it was not used as widely as possible, even when treating prostitutes in the London Lock Hospital. Aware of the objections against the instrument, he urged its use:

'Every venereal prostitute should be examined with the speculum for her own sake and that of her medical attendant. To attempt her cure without it is to waste the time of both parties.'

The very lack of facilities may be another reason to explain the lack of progress made in English venereology. The British and Foreign Medico-Chirurgical Review (1851), at the time of the Great Exhibition, was 'at a loss to point out what science has gained by the writings of the London surgeons on syphilis, since the commencement of the present century'. London had vastly superior facilities for research compared with other English towns but they were inferior to those of other European countries. In France, Belgium, Prussia, Austria, and Denmark, there was legislation controlling prostitution which resulted in the compulsory hospitalization and treatment of prostitutes, and this might have provided facilities for more systematic observation than was possible in English hospitals. Certainly, when Britain imitated the continental legislation and introduced the Contagious Diseases Acts (1864-69), apart from the expected decline of venereal disease in the military population, the medical profession hoped that increased research would erase some of the question marks which hung over the subject.

Although in these years venereology did not experience advances comparable with those associated with microbiology in the closing decades of the century, there was a perceptible widening of the frontier of knowledge, chiefly through the impetus of continental research. In England the government enquiry indicates that, while some advances had been achieved, there was still vagueness and uncertainty in pathology, diagnosis, and treatment, and a general absence of serious scientific research. The subject was still wrapped in mystery. This situation will not be understood unless it is recognized that the doctors who were at the frontier were likely to be unrepresentative of their profession. The researchers experimented and published their findings but whether the new techniques were put into practice by their colleagues is unknown. Perhaps English doctors preferred the traditional methods and prescribed the blue pill upon the appearance of possible venereal symptoms. It is more difficult to assess changes in attitudes than changes in medical practice.

Discussion was open in the medical periodicals, and after the passing of the Contagious Diseases Acts in the general press, but there was still the social stigma. Changes in treatment were certainly hindered by questions of morality, and because of this the 
venereal diseases were in a sense distinct and not open to the same scientific procedures as other diseases. But a relaxation of attitudes does appear to have occurred on the part of some hospitals who were willing to treat and admit venereal cases. In this area of social medicine, to borrow the lines of Brecht, we are left with

So many particulars

So many questions.

\section{Summary}

Hospital facilities for treating venereal disease in England are outlined and the diagnosis and treatment of syphilis and gonorrhoea between 1800 and 1870 is discussed. Some reasons for the relatively slow development of venereology in England are suggested.

\section{References}

ABeRnETHy, J. (1810) 'Surgical Observations on Diseases resembling Syphilis, and on Diseases of the Urethra'. Longman Hurst, Rees and Orme, London

Acton, W. (1857) 'Prostitution, considered in its Moral, Social and Sanitary Aspects, in London and other Large Cities with Proposals for the Mitigation and Prevention of its Attendant Evils'. Churchill, London

Balbirnie, J. (1836) 'The Speculum applied to the Diagnostic and Treatment of the Organic Diseases of the Womb'. Longmans, London

BoERHAAVE, H. (1709) 'Aphorismi', trans. from the last edition printed in Latin at Leyden, 1722, as 'Boerhaave's Aphorisms Concerning the Knowledge and Cure of Diseases', p. 439. (London, 1724)

British and Foreign Medical Review (1840a) 10, 400

- (1840b) 10, 392

British and Foreign Medico-Chirurgical Review (1851), 8, 203

$-(1857 \mathrm{a})$ 19, 412
$-(1857 \mathrm{~b}) 19,415$
$-(1865) 35,47$
- (1869a) 44, 80
$-(1869 \mathrm{~b}) \mathbf{4 4}, 88$

DunCan, A. (1772) 'Observations on the Operation and Use of Mercury in the Venereal Disease'. Edinburgh

Fergusson, W. (1813) Med.-chir. Trans., 4, 1

GuTHRIE, G. J. (1817) Ibid., 8, 550

Hamilton, J. (1819) 'Observations on the Use and Abuse of Mercurial Medicines in Various Diseases'. Constable, Edinburgh

HanNay, A. (1837) Lond. med. Gaz., 20, 187

Holland, T. S. (1854) Brit. For. med.-chir. Rev., 13, 457
LANE, J. R., and Gascoyen, G. C. (1867) Med.-Chir. Trans., 50, 281

LANCEREAUx, E. (1869) 'A Treatise on Syphilis, Historical and Practical', vol. 2, trans. G. Whitley. New Sydenham Society, London

LEE, H. (1863) 'Lectures on Syphilitic and VaccinoSyphilitic Inoculation', 2nd ed. Churchill, London

Mathias, A. (1810) 'The Mercurial Disease ... with Observations on its Connexion with Lues Venerea'. London

Medico-Chirurgical Review (1837) 26, 177

Pearson, J. (1800) 'Observations on the Effects of Various Articles of the Materia Medica in the Cure of Lues Venerea. . . Callow, London

PusEy, W. A. (1933) 'The History and Epidemiology of Syphilis'. Thomas, Springfield, Ill.

Report (1864) 'Report by Dr. J. S. Bristowe and Mr. T. Holmes on the Hospitals of the United Kingdom', in Sixth Report of the Medical Officer of Health to the Privy Council for 1863. Cd. (3416). Appendix 15, p. 463

Report (1868) 'Report of the Committee Appointed to Enquire into the Pathology and Treatment of the Venereal Disease, with the View to Diminish its Injurious Effects on the Men of the Army and Navy ... 1867-68'. Cd. (4031)

The Committee sat under the chairmanship of F. C. Skey from 1864 to 1865 and its final report was published in 1868

Ricord, P. (1842) 'A Practical Treatise on Venereal Disease', trans. H. P. Drummond. Longman, Brown, Green, and Longmans, London

Rose, T. (1817) Med-chir. Trans., 8, 349

WAGSTAFFE, W. W. (1869) 'The quantity and kinds of venereal disease under treatment at certain charitable institutions in London', in Eleventh Report of the Medical Officer of Health to the Privy Council for 1868. Cd. (4127). Appendix 4, p. 78

WALLACE, W. (1836) Lancet, 2, 5

Waugh, M. A. (1971) Brit. F. vener. Dis., 47, 146

WILliaMS, R. (1841) 'Elements of Medicine', vol. 2: 'On Morbid Poisons'. Baillière, London

\section{Facilités hospitalières dans le diagnostic et le traitement des maladies vénériennes en Angleterre de 1800 à 1870}

\section{SOMMAIRE}

On expose l'état des facilités hospitalières dans le traitement des maladies vénériennes en Angleterre, et l'on discute du diagnostic et du traitement de la syphilis et de la gonococcie entre 1800 et 1870 . On avance quelques raisons pour expliquer le développement relativement lent de la vénéréologie en Angleterre. 